\title{
Effects of Reordering and Lumping in the Analysis of Discrete-Time SANs
}

TuĞRul DaYAR ${ }^{1}$ Department of Computer Engineering, Bilkent University, 06533 Bilkent, Ankara, Turkey. tugrul@cs.bilkent.edu.tr

\begin{abstract}
In a recent paper [13], it is shown that discrete-time stochastic automata networks (SANs) are lumpable under rather general conditions. Therein, the authors present an efficient iterative aggregation-disaggregation (IAD) algorithm geared towards computing the stationary vector of discrete-time SANs that satisfy the conditions of lumpability. The performance of the proposed IAD solver essentially depends on two parameters. The first is the order in which the automata are lined up, and the second is the size of the lumped matrix. Based on the characteristics of the SAN model at hand, the user may have some flexibility in the choice of these two parameters. In this paper, we give rules of thumb regarding the choice of these parameters on a model from mobile communications.
\end{abstract}

Key words. Stochastic automata networks, discrete-time Markov chains, lumpability, iterative aggregation-disaggregation

\section{Introduction}

Stochastic Automata Networks (SANs) $[19,20,22,21,23,11,25,12,26,6,2$, $4,9,27,28,3,10,13]$ comprise a methodology for modeling Markovian systems that have interacting components. The methodology is based on decomposing the system to be investigated into its components and modeling each component independently. Afterwards, interactions and dependencies among components are introduced and the model finalized. The two advantages of SANs that result from this divide-and-conquer approach are the following. Each component can be modeled much easier compared to the global system due to state space reduction. Storage space allocated for components is minimal compared to the case in which transitions from each global state are stored explicitly. However, all this comes at the expense of longer analysis time $[12,26,2,6,4,9,27,3,13]$.

A discrete-time system of $N$ components can be modeled by a single stochastic automaton for each component. With this decompositional approach, the global system ends up having as many states as the product of the number of states of the individual components. See [25, Ch. 9] for detailed information regarding SANs. When there are $E$ synchronizing events in the system, automaton $k$ denoted by $\mathcal{A}^{(k)}$ has the corresponding transition probability matrix $P_{e}^{(k)}$ that represents the contribution of $\mathcal{A}^{(k)}$ to synchronization $e \in\{0,1, \ldots, E-1\}$ (see [10, p. 333]). For convenience, we number the automata and synchronizing events starting from 0 . The underlying discrete-time MC (DTMC) corresponding to the global system can be obtained from

$$
P=\sum_{e=0}^{E-1} \bigotimes_{k=0}^{N-1} P_{e}^{(k)}
$$

\footnotetext{
${ }^{1}$ This work is supported by TÜBITTAK-CNRS grant.
} 
We refer to the tensor representation in equation (1) associated with the DTMC as the descriptor of the SAN. Assuming that $\mathcal{A}^{(k)}$ has $n_{k}$ states, the global system has $n=\prod_{k=0}^{N-1} n_{k}$ states. When there are transition probabilities in $P_{e}^{(k)}$ that are functions of the global state of the system rather than only $\mathcal{A}^{(k)}$, tensor products become generalized tensor products [23]. We consider the form of the descriptor in equation (1) rather than the one in [19] since it is compact and easier to work with.

The difficulty associated with discrete-time SANs is that the matrices $P_{e}^{(k)}$ are relatively dense compared to their continuous-time counterparts implying a larger number of floating-point arithmetic operations in the generalized descriptorvector multiply algorithm (see $[9$, p. 404]) used in iterative solvers. Generally, the underlying DTMC of a discrete-time SAN is a dense matrix as opposed to the generator corresponding to a continuous-time SAN. Therefore, discrete-time SANs can be used to tackle even small systems composed of interacting components but that have dense DTMCs since the underlying MC is not generated and stored during SAN analysis.

In the next section, we provide information about the wireless ATM model that is investigated. In the third section, we discuss the results in [13] related to ordering and lumping of automata. In the fourth section, we provide results of numerical experiments, and in the fifth section, we conclude.

\section{The model}

The application considered in [13] is a multiservices resource allocation policy (MRAP) that integrates three types of service over a time division multiple access (TDMA) system in a mobile communication environment. We have the constant bit rate (CBR) service for two types of voice calls (i.e., handover calls from neighboring cells and new calls), the variable bit rate (VBR) service for two types of calls as in CBR service, and the available bit rate (ABR) service for data transfer. A single cell and a single carrier frequency is considered, and the system is modeled as a discrete-time SAN in which state changes occur at TDMA frame boundaries. Regarding the events that take place in the system, the following assumptions are made. Data packet and call arrivals to the system happen at the beginning of a frame, and data packet transmissions finish and calls terminate at the end of the frame. Since each data packet is small enough to be transmitted in a single slot of a TDMA frame, in a particular state of the system it is not possible to see slots occupied by data packets.

Now, let us move to the parameters of the model. Data is queued in a FIFO buffer of size $B$ and has the least priority. The arrival of data packets is modeled as an on-off process. The process moves from the on state to the off state with probability $\alpha$ and vice versa with probability $\beta$. The load offered to the system is defined as $\lambda=\beta /(\alpha+\beta)$. Assuming that the time interval between two consecutive on periods is $t$, the burstiness of such an on-off process is described by the square coefficient of variation, $S_{C}=\operatorname{Var}(t) /[E(t)]^{2}$. In terms of $\lambda$ and $S_{C}$, $\beta=2 \lambda(1-\lambda) /\left(S_{C}+1-\lambda\right)$ and $\alpha=\beta(1-\lambda) / \lambda$. When the on-off process is in the 
on state, we assume that $i \in\{0,1,2,3\}$ data packets may arrive with probability $p_{d i}$. The mean arrival rate of data packets is defined as $\rho=\sum_{i=1}^{3} i \times p_{d i}$. Hence, the global mean arrival rate of data packets is given by $\Gamma=\lambda \rho$. If the number of arriving data packets exceeds the free space in the buffer plus the number of free slots in the current TDMA frame, the excess packets are dropped.

Handover CBR requests have priority over new CBR calls and they respectively arrive with probabilities $p_{h}$ and $p_{n}$. Similarly, the probabilities of VBR new call and handover arrivals during a TDMA frame have geometrical distributions with parameters $p_{v n}$ and $p_{v h}$, respectively. We do not consider multiple handover or new CBR/VBR call arrivals during a TDMA frame since the associated probabilities with these events are small. Each CBR/VBR call takes up a single slot of a TDMA frame but may span multiple TDMA frames. When all the slots are full, incoming CBR/VBR calls are rejected. The number of CBR calls that may terminate in a TDMA frame depends on the number of active CBR calls, but can be at most $M$, and hence is modeled as a truncated binomial process with parameter $p_{s}$.

On the other hand, a VBR connection is characterized by a state of high intensity and a state of low intensity. In the former, the VBR source transmits data with its peak rate, whereas in the latter, its transmission rate is lower. The reduced transmission rate in the low intensity state in fact means that at some instances of time the slot in a TDMA frame allocated to the VBR connection is not used. A VBR connection moves from the high intensity state to the low intensity state with probability $\alpha_{v}$ and vice versa with probability $\beta_{v}$. In terms of $\lambda_{v}=\beta_{v} /\left(\alpha_{v}+\beta_{v}\right)$ and its square coefficient of variation $S_{C v}$, we have $\beta_{v}=2 \lambda_{v}\left(1-\lambda_{v}\right) /\left(S_{C v}+1-\lambda_{v}\right)$ and $\alpha_{v}=\beta_{v}\left(1-\lambda_{v}\right) / \lambda_{v}$. When in the low intensity state, the VBR connection moves from the case of a busy slot to the case of an empty slot with probability $p_{\text {empty }}$ and vice versa with probability $p_{\text {busy }}$. State changes of a VBR connection after it is set up are assumed to take place at the end of a TDMA frame. We further assume that when a VBR connection is set up as either a new call or a handover, it is in the high intensity state. However, the connection can terminate in any state of the VBR source. We also assume that when a VBR connection changes its state from high intensity to low intensity, it enters the state with a busy slot. The number of VBR calls that may terminate in a TDMA frame depends on the number of active VBR calls and the duration of each VBR call is assumed to be a geometric process with parameter $p_{v s}$.

Each TDMA frame that gives CBR, VBR, and ABR service consists of $C$ slots reserved for CBR traffic and $V$ slots reserved for VBR traffic. ABR traffic can be pushed into any reserved, but unused slots. Hence, data packets can be transmitted in the idle slots among the $C$ reserved for CBR traffic, in the idle slots among the $V$ reserved for VBR traffic, and in those slots among the $V$ that are in the low intensity state but are empty. The SAN model consists of $(3+V)$ automata and 9 synchronizing events. States of all automata are numbered starting from 0 . We denote the state index of automaton $k$ by $s \mathcal{A}^{(k)}$. Automaton $\mathcal{A}^{(0)}$ represents the data arrival process and has two states that correspond to the on and off states of the data source. Transitions in this automaton happen independently of other automata. Automaton $\mathcal{A}^{(1)}$ represents the portion of the TDMA frame reserved 
for CBR calls and has $(C+1)$ states. Automaton $\mathcal{A}^{(2)}$ represents the data buffer and has $(B+1)$ states, where $B$ is the buffer size. Transitions of this automata depend on $\mathcal{A}^{(0)}$ and $\mathcal{A}^{(1)}$. Automata $\mathcal{A}^{(3)}$ through $\mathcal{A}^{(2+V)}$ represent the $V$ slots reserved for VBR traffic. Each automaton corresponding to VBR traffic has four states. State 0 of the automaton corresponds to the case of an idle slot, i.e., the VBR connection is not active. State 1 corresponds to the state of high intensity state, states 2 and 3 correspond to the state of low intensity. Particularly, state 2 indicates that the slot is busy and state 3 indicates that it is empty. Each automaton $\mathcal{A}^{(k)}, k \in\{4,5, \ldots, V+2\}$, that models VBR traffic depends on the automata $\mathcal{A}^{(3)}, \mathcal{A}^{(4)}, \ldots, \mathcal{A}^{(k-1)}$ since we assume that arriving VBR calls are dispatched to slots starting from the smallest indexed VBR automaton. We remark that the automata of the SAN that handle CBR and VBR arrivals are mutually independent. Hence, the set of synchronizing events are given by Cartesian product, and $e_{a b}$ denotes the synchronizing event that is triggered by $a \mathrm{CBR}$ arrivals and $b \mathrm{VBR}$ arrivals for $a, b \in\{0,1,2\}$.

The probability matrices associated with the automata are all relatively dense except the ones that correspond to the data buffer when $s \mathcal{A}^{(0)}=0$. See [13] for a detailed description of the system. In passing, we remark that the discrete-time SAN model of the system has a global state space size of $n=2(C+1)(B+1) 4^{V}$. For the problem with $(C, V, B)=(8,2,15), \lambda=0.1, S_{C}=1,\left(p_{d 0}, p_{d 1}, p_{d 2}, p_{d 3}\right)=$ $(0.05,0.1,0.25,0.6)$ (amounting to an average of $\rho=2.5$ packet arrivals during a TDMA frame), $\left(p_{n}, p_{h}, p_{s}\right)=C\left(5 \times 10^{-6}, 10^{-5}, 5 \times 10^{-6}\right), \lambda_{v}=0.5, S_{C v}=10$, $\left(p_{\text {empty }}, p_{\text {busy }}\right)=(0.9,0.1),\left(p_{v n}, p_{v h}, p_{v s}\right)=V\left(5 \times 10^{-6}, 10^{-5}, 5 \times 10^{-6}\right)$, and at most $2(=M)$ CBR departures during a TDMA frame, we have $n=4,608$ and $n z=1,618,620$ (number of nonzeros larger than $10^{-16}$ is $1,174,657$ ). Here $n z$ denotes the number of nonzeros in the underlying DTMC.

We refer to the rejection of an existing call as dropping and to the rejection of a new call or packet as blocking. The performance measures of interest are the dropping probabilities of handover CBR and handover VBR calls, the blocking probabilities of new CBR calls and new VBR calls, and the blocking probability of data packets. Once the steady state vector of the descriptor is computed, each of the performance measures may be determined [13]. In this work, the aim is not to present values of performance measures for a set of parameters, but rather is to discuss the implications on the IAD solver of reordering the automata and choosing the size of the lumped matrix. Therefore, we constrain ourselves to reporting solution times and iteration counts in section 4. Now, let us summarize the theoretical results in [13].

\section{$3 \quad$ Reordering and Lumping}

The automata of some discrete-time SANs can be reordered and then renumbered so that transitions of $\mathcal{A}^{(k)}$ for $k \in\{1,2, \ldots, N-1\}$ depend (if at all) on the states of the lower indexed automata $\mathcal{A}^{(0)}, \mathcal{A}^{(1)}, \ldots, \mathcal{A}^{(k-1)}$ (the functional dependency being represented by $\mathcal{A}^{(k)}\left[\mathcal{A}^{(0)}, \mathcal{A}^{(1)}, \ldots, \mathcal{A}^{(k-1)}\right]$ ) (see [9] for details). Note that there must exist at least one automaton (in our case, it is $\mathcal{A}^{(0)}$ ) that is independent of all 
the other automata for such an ordering to be possible. In our model, such an ordering of automata is possible, and it implies that the automaton $\mathcal{A}^{(2)}$ be placed in the last position and the automata $\mathcal{A}^{(k)}, k \in\{3,4, \ldots, V+2\}$, be placed in any position other than the last as long as they are ordered according to increasing index among themselves. Hence, one possibility is $\mathcal{A}^{(0)}, \mathcal{A}^{(1)}, \mathcal{A}^{(3)}, \mathcal{A}^{(4)}, \ldots, \mathcal{A}^{(V+2)}, \mathcal{A}^{(2)}$.

Now, let us assume that the automata are reordered as described and renumbered from 0 to $(N-1)$. To such an ordering of automata correspond block partitionings of the form

$$
P_{n \times n}=\left(\begin{array}{cccc}
P_{11} & P_{12} & \ldots & P_{1 K} \\
P_{21} & P_{22} & \ldots & P_{2 K} \\
\vdots & \vdots & \ddots & \vdots \\
P_{K 1} & P_{K 2} & \ldots & P_{K K}
\end{array}\right)
$$

in which all the blocks $P_{i j}$ are square, of order $\prod_{k=m}^{N-1} n_{k}$, and $K=\prod_{k=0}^{m-1} n_{k}$ for any $m \in\{1,2, \ldots, N-1\}$. Hence, for the given ordering of automata, there are $(N-1)$ different such partitionings which vary between one that has $n_{0}$ diagonal blocks of order $\prod_{k=1}^{N-1} n_{k}$ and one that has $\prod_{k=0}^{N-2} n_{k}$ diagonal blocks of order $n_{N-1}$. Each of the $(N-1)$ block partitionings is lumpable as stated in the next theorem of [13].

Theorem 1 A discrete-time $S A N$ of $N$ automata and $E$ synchronizing events whose automata are reordered and renumbered so that $\mathcal{A}^{(k)}\left[\mathcal{A}^{(0)}, \mathcal{A}^{(1)}, \ldots, \mathcal{A}^{(k-1)}\right]$, $k \in\{1, \ldots, N-1\}$, and that has the descriptor in equation (1) with equal row sums in each $P_{e}^{(k)}$ for $k=0,1, \ldots, N-1$ and $e=0,1, \ldots, E-1$ is lumpable with respect to the partitioning in equation (2) for any $m \in\{1,2, \ldots, N-1\}$.

Now, we state the more relaxed version of Theorem 1 in [13] for the case of cyclic functional dependencies.

Definition 1 Let $G(\mathcal{V}, \mathcal{E})$ be the directed graph (digraph) corresponding to a discrete-time $S A N$ in which the vertex $v_{k} \in \mathcal{V}$ represents $\mathcal{A}^{(k)}$ and the edge $\left(v_{k}, v_{l}\right)$ $\in E$ if transitions in $\mathcal{A}^{(k)}$ depend on the state of $\mathcal{A}^{(l)}$ (i.e., $\left.\mathcal{A}^{(k)}\left[\mathcal{A}^{(l)}\right]\right)$. Then the $S A N$ is said to contain cyclic functional dependencies if and only if the digraph has at least one strongly connected component (SCC) composed of multiple automata.

Detailed description of the SCC algorithm for digraphs can be found in [1, pp. 191-197].

Theorem $2 A$ discrete-time $S A N$ of $N$ automata $\mathcal{A}^{(k)}, k=0,1, \ldots, N-1$, and $E$ synchronizing events that contains cyclic functional dependencies among its automata is lumpable if the digraph corresponding to the SAN has more than one $S C C$ and each $P_{e}^{(k)}$ has equal row sums for $k=0,1, \ldots, N-1$ and $e=0,1, \ldots$, $E-1$.

Assuming that $P$ is lumpable with respect to the partition in (2) and is irreducible, in [13] the following modified form of Koury-McAllister-Stewart's IAD 
algorithm [15] is proposed for computing the stationary probability vector $\pi$ (i.e., $\pi P=\pi,\|\pi\|=1)$. The convergence analysis of the KMS algorithm is based on the concept of near complete decomposability (NCD) [18]. In [16] and [24], it is shown that fast convergence is achieved if the degree of coupling, $\|F\|_{\infty}$, is small compared to 1 , where $P=F+\operatorname{diag}\left(P_{11}, P_{22}, \ldots, P_{K K}\right)$.

\section{Algorithm 1. IAD algorithm for discrete-time SANs}

1. Let $\pi^{(0)}=\left(\pi_{1}^{(0)}, \pi_{2}^{(0)}, \ldots, \pi_{K}^{(0)}\right)$ be a given initial approximation of $\pi$. Set $i t=1$.

2. Aggregation:

(a) Compute the lumped matrix $L$ of order $K$ with $i j$ th element $l_{i j}=\max \left(P_{i j} u\right)$.

(b) Solve the singular system $\tau(I-L)=0$ subject to $\|\tau\|_{1}=1$ for $\tau=\left(\tau_{1}, \tau_{2}, \ldots, \tau_{K}\right)$.

3. Disaggregation:

(a) Compute the row vector

$$
z^{(i t)}=\left(\tau_{1} \frac{\pi_{1}^{(i t-1)}}{\left\|\pi_{1}^{(i t-1)}\right\|_{1}}, \tau_{2} \frac{\pi_{2}^{(i t-1)}}{\left\|\pi_{2}^{(i t-1)}\right\|_{1}}, \cdots, \tau_{K} \frac{\pi_{K}^{(i t-1)}}{\left\|\pi_{K}^{(i t-1)}\right\|_{1}}\right) .
$$

(b) Solve the $K$ nonsingular systems of which the $i$ th is given by

$$
\pi_{i}^{(i t)}\left(I-P_{i i}\right)=b_{i}^{(i t)}
$$

for $\pi_{i}^{(i t)}, i=1,2, \ldots, K$, where

$$
b_{i}^{(i t)}=\sum_{j>i} z_{j}^{(i t)} P_{j i}+\sum_{j<i} \pi_{j}^{(i t)} P_{j i} .
$$

4. Test $\pi^{(i t)}$ for convergence. If the desired accuracy is attained, then stop and take $\pi^{(i t)}$ as the stationary probability vector of $P$. Else set $i t=i t+1$ and go to step 3 .

In [17], the convergence of a framework of IAD methods is studied. The IAD algorithm considered is different in that there is no requirement of NCDness on the partitioning. Furthermore, a number of relaxations (i.e., smoothings) of the power method kind is performed at the fine level. The authors prove that the errors at the fine and coarse levels are intimately related, and for a strictly positive initial approximation, the IAD approximation converges rapidly to the stationary vector as long as one is very precise in computing at the coarse level and a sufficiently high number of smoothings is performed at the fine level. Numerical results on randomly generated stochastic matrices with varying degrees of coupling and having equal 
orders of blocks which are all tridiagonal show that convergence is practically independent of the degree of coupling.

The motivation behind proposing Algorithm 1 rather than block Gauss-Seidel (BGS) for discrete-time SANs is that the partitioning in (2) is a balanced one with equal orders of blocks and the aggregate matrix needs to be formed only once due to lumpability. See [8] for recent results on the computation of the stationary vector of Markov chains.

In Algorithm 1, the lumped matrix $L$ of order $K=\prod_{k=0}^{m-1} n_{k}$ is computed at the outset and solved once for its stationary vector $\tau$. Note that the lumped matrix $L$ is also lumpable if $m>1$. These hint at the solver to be chosen at step 2 to compute $\tau$. Assuming that $L$ is dense, one may opt for a direct solver such as Gaussian elimination (GE) (or the method of Grassmann-Taksar-Heyman, $\mathrm{GTH}$, if $L$ is relatively ill-conditioned) when $K$ is on the order of hundreds. Else one may use IAD with a lumped matrix of order $\prod_{k=0}^{m^{\prime}-1} n_{k}$, where $1<m^{\prime}<m$ (or IAD with an NCD partitioning if $L$ is relatively ill-conditioned). In any case, sufficient space must be allocated to store $L$. As for the disaggregation phase (i.e., a BGS iteration), the right-hand sides $b_{i}^{(i t)}$ at iteration it may be computed efficiently as shown in [13] at the cost of $E(K-2)$ vectors of length $\prod_{k=m}^{N-1} n_{k}$, that is roughly $E$ vectors of length $n$. In summary, the proposed solver is limited by $\max \left(K^{2},(E+2) n\right)$ amount of double precision storage assuming that the lumped matrix is stored in two dimensions. The 2 vectors of length $n$ are used to store the previous and current approximations of the solution.

So far, we have assumed that the underlying DTMC of the given discretetime SAN is irreducible. Since this may not be the case, a state classification (SC) algorithm that partitions the global state space of a SAN into essential and transient subsets is implemented [13]. The inhibition of transient states is important in removing redundant computation from the IAD solver. However, the case of more than one partition of essential states is not considered since it hints at a modeling problem.

Now, let us return to the model in section 2 and consider lumpable orderings of the automata. Since $\mathcal{A}^{(2)}$ must be the last automaton in a lumpable ordering, there are $(V+1)$ ! ways in which $\mathcal{A}^{(0)}$ may precede $\mathcal{A}^{(1)}$ among the first $(V+2)$ automata. The same argument is true of $\mathcal{A}^{(0)}$ succeeding $\mathcal{A}^{(1)}$ among the first $(V+2)$ automata. Therefore, the requirement in Theorem 1 regarding functional dependencies implies that there are $2(V+1)$ ! orderings that may be used. Hence, we have 12 lumpable orderings to choose from when $V=2$ and 48 lumpable orderings to choose from when $V=3$. Since these are large numbers of orderings to investigate, we treat the VBR automata as a single entity and concentrate on only the 3 ! orderings of automata given by $[(3,4, \ldots, V+2), 1,0,2]$, $[1,(3,4, \ldots, V+2), 0,2], \quad[(3,4, \ldots, V+2), 0,1,2], \quad[0,(3,4, \ldots, V+2), 1,2]$, $[1,0,(3,4, \ldots, V+2), 2]$, and $[0,1,(3,4, \ldots, V+2), 2]$. We name these orderings respectively $o_{1}, o_{2}, \ldots, o_{6}$. Regarding the lumping parameter, $m$, we choose values from the set $\{V-1, V, V+1\}$ which implies that the largest lumped matrix formed in step 2 is of order $2(C+1) 4^{V}$ and the smallest system to solve in step 3 is of order $(B+1)$. 
We remark that a reordering of discrete-time automata corresponds to a symmetric permutation of the underlying DTMC. In other words, reordering of automata is equivalent to a renumbering of the global states in a SAN model. Since the objective of this study is to investigate the effects of reordering and the lumping parameter, $m$, on the convergence of Algorithm 1, we choose the integer parameters $(C, V, B)$ so that the lumped matrix formed in step 2 can be solved accurately and rapidly using the GTH method as discussed in [7] and there is sufficient space to factorize in sparse format the $K$ diagonal blocks in step 3(b) at the outset. Hence, we use sparse forward and back substitutions to solve the $K$ nonsingular systems at each iteration of Algorithm 1 and do not need to employ any smoothings.

\section{Numerical results}

Algorithm 1 is implemented in $\mathrm{C}++$ as part of the software package PEPS [22]. We time the solver on a Pentium III with 64 MBytes of RAM under Linux. In each experiment, we use a tolerance of $10^{-8}$ on the approximate error $\left\|\pi^{(i t)}-\pi^{(i t-1)}\right\|_{2}$ in step 4 of Algorithm 1 . The approximate residual $\left\|\pi^{(i t)}-\pi^{(i t)} P\right\|_{2}$ turns out to be less than the approximate error upon termination in all our experiments.

In the first set of experiments, we consider three variants of the problem $(C, V, B)=(8,2,15)$, which has a state space size of $n=4,608$ (see section 2). We set $\left(p_{d 0}, p_{d 1}, p_{d 2}, p_{d 3}\right)=(0.05,0.1,0.25,0.6), \lambda_{v}=0.5, S_{C v}=10,\left(p_{\text {empty }}, p_{\text {busy }}\right)=$ $(0.9,0.1)$ in the first variant and $\left(p_{d 0}, p_{d 1}, p_{d 2}, p_{d 3}\right)=(0.4,0.3,0.2,0.1), \lambda_{v}=0.5$, $S_{C v}=1,\left(p_{\text {empty }}, p_{\text {busy }}\right) \in\{(0.9,0.1),(0.5,0.5)\}$ in the last two variants. The other parameters are chosen as $M=V, S_{C}=1, \lambda \in\{0.1,0.3,0.5,0.7,0.9\},\left(p_{n}, p_{h}, p_{s}\right)=$ $C\left(5 \times 10^{-6}, 10^{-5}, 5 \times 10^{-6}\right)$, and $\left(p_{v n}, p_{v h}, p_{v s}\right)=V\left(5 \times 10^{-6}, 10^{-5}, 5 \times 10^{-6}\right)$. The lumpability parameter assumes the values in $\{2,3,4\}$. The degree of coupling, $\|F\|_{\infty}$, associated with the partitioning in equation (2) for each DTMC is on the order of $10^{-1}$ and mostly close to 1.0. Hence, the lumpable partitionings we consider in Algorithm 1 for the first set of experiments are not NCD. However, the smallest degree of coupling we find for each DTMC using the algorithm in [5] is on the order of $10^{-5}$. All this means that even though the lumpable partitionings we consider are not NCD partitionings, there exist highly NCD partitionings for each DTMC, and therefore they are all very ill-conditioned.

The underlying DTMCs of the SAN models in the first set of experiments are reducible with a single subset of essential states and 224 transient states. It is argued in [13] that when a reducible discrete-time SAN has a single subset of essential states and each subset of the partition in equation (2) includes at least one essential state (which is the case in our experiments), the lumped matrix computed in step 2 of Algorithm 1 is irreducible. With such a partitioning, if one starts in step 1 with an initial approximation having zero elements corresponding to transient states, successive computed approximations will have zero elements corresponding to transient states as well. In our experiments, we start with a positive initial approximation and observe that all elements that correspond to transient states become zero at the second iteration. Once the elements that cor- 
respond to transient states in an approximate solution become zero, they remain zero. Hence, there is no need to run the time consuming SC algorithm for each of the experiments, since the matrices in each of the three variants have the same nonzero structure.

In the first variant, Algorithm 1 converges for the orderings $o_{1}$ and $o_{2}$ within 34 iterations when $m \in\{3,4\}$, whereas for the other four orderings it converges within 34 iterations only when $m=4$. In all other cases, Algorithm 1 does not converge within 250 iterations to the prespecified tolerance. A similar observation follows for the second and third variants if we replace the 34 respectively with 65 and 120 (achieved in $o_{4}-O_{6}$ ). Hence, the three variants of this problem seem to be of increasing difficulty as we move from the first to the third, although each one has highly NCD partitionings with degree of coupling on the same order of $10^{-5}$. In Table 1, we present the results of numerical experiments with Algorithm 1 for the three variants using $o_{1}$. Note that a smaller number of iterations may not imply a smaller solution time as in the first variant for $\lambda=0.9$. The best solution times are obtained with $o_{1}$ (and $o_{2}$ ) when $m=3$ is used. For each of the six orderings, $m=4$ gives a lumped matrix of order $K=288$. For $o_{1}$ and $o_{2}, m=3$ gives a lumped matrix of order $K=144$. For the other four orderings, $m=3$ gives lumped matrices of orders 32 and 72, which are both smaller than 144. For the six orderings, $m=2$ gives lumped matrices of orders varying between 8 and 36 . It takes nearly 0 seconds to solve the lumped matrix using GTH in all cases. Hence, the timing results in Table 1 are for the iterative part of Algorithm 1.

TABLE 1. Solution times in seconds and \# of iterations with $o_{1}$ for 1st set of experiments.

\begin{tabular}{|c|c||rc|rc|rr|rr|rr|}
\hline Variant & \multicolumn{1}{|c||}{$m$} & \multicolumn{2}{c|}{$\lambda=0.1$} & \multicolumn{2}{c|}{$\lambda=0.3$} & \multicolumn{2}{c|}{$\lambda=0.5$} & \multicolumn{2}{|c|}{$\lambda=0.7$} & \multicolumn{2}{|c|}{$\lambda=0.9$} \\
& & Time & \#it & Time & \#it & Time & \#it & Time & \#it & Time & \#it \\
\hline \hline \multirow{2}{*}{1} & 4 & 25 & 28 & 30 & 34 & 27 & 30 & 23 & 26 & 22 & 24 \\
& 3 & 8 & 18 & 9 & 21 & 8 & 17 & 9 & 20 & 12 & 27 \\
& 2 & 84 & $250^{+}$ & 84 & $250^{+}$ & 84 & $250^{+}$ & 84 & $250^{+}$ & 84 & $250^{+}$ \\
\hline 2 & 4 & 48 & 51 & 52 & 56 & 41 & 44 & 35 & 38 & 61 & 65 \\
& 3 & 22 & 48 & 22 & 47 & 17 & 38 & 17 & 37 & 27 & 61 \\
& 2 & 84 & $250^{+}$ & 84 & $250^{+}$ & 84 & $250^{+}$ & 84 & $250^{+}$ & 84 & $250^{+}$ \\
\hline 3 & 4 & 111 & 118 & 53 & 57 & 51 & 55 & 44 & 47 & 84 & 90 \\
& 3 & 51 & 114 & 23 & 52 & 22 & 49 & 20 & 45 & 40 & 89 \\
& 2 & 84 & $250^{+}$ & 84 & $250^{+}$ & 84 & $250^{+}$ & 84 & $250^{+}$ & 84 & $250^{+}$ \\
\hline
\end{tabular}

In the second set of experiments, we consider the problem $(C, V, B)=(3,3,15)$, which has a state space size of $n=8,192$. We set $\left(p_{d 0}, p_{d 1}, p_{d 2}, p_{d 3}\right)=$ $(0.05,0.1,0.25,0.6), \lambda_{v}=0.5, S_{C v}=10,\left(p_{\text {empty }}, p_{\text {busy }}\right)=(0.9,0.1)$, the other parameters being chosen as in the first set of experiments. The lumpability parameter assumes the values in $\{3,4,5\}$. Again, even though the lumpable partitionings we consider are not NCD partitionings, there exist highly NCD partitionings for each DTMC, and therefore they are all very ill-conditioned. The underlying DTMCs of the SAN models in the second set of experiments are irreducible. 
Algorithm 1 converges for the orderings $o_{1}$ and $o_{2}$ within 42 iterations when $m \in\{4,5\}$, whereas for the other four orderings it converges within 43 iterations (achieved in $o_{4}-o_{6}$ ) only when $m=5$. In all other cases, Algorithm 1 does not converge within 250 iterations to the prespecified tolerance. The best solution times are obtained with $o_{1}$ and $o_{2}$ when $m=4$ is used.

Regarding the ordering of automata, $o_{1}$ and $o_{2}$ are more advantageous than the other four orderings since they converge for more values of $m$. The orderings $o_{1}$ and $o_{2}$ have $\mathcal{A}^{(0)}$ and $\mathcal{A}^{(2)}$ as the last two automata. These automata have transition probabilities of the same order. Furthermore, when $m=N-2$ is used with $o_{1}$ and $o_{2}$, the lumped matrix is larger than one would have with the other four orderings. We believe these two factors influence the behavior of Algorithm 1 for $o_{1}$ and $o_{2}$ when $m=N-2$. Further experiments must be conducted to improve our confidence in this conjecture.

\section{Conclusion}

Experiments on a problem from mobile communications indicate that the performance of Algorithm 1 for discrete-time SANs is sensitive to the ordering of automata and the choice of the lumpability parameter. Even though the coarse and fine level solutions are computed with high accuracy in our experiments, there are values of the lumpability parameter for which fast convergence of Algorithm 1 is not witnessed. On the other hand, some partitionings converge in a smaller number of iterations than others. The value of the lumpability parameter determines the order of the coupling matrix. Numerical results imply that convergence may not be observed in a reasonable number of iterations when the order of the coupling matrix is smaller than the squareroot of the state space size. Hence, it is recommended for one to consider the largest permissible value of the lumpability parameter when using Algorithm 1.

Acknowledgment. The author thanks Oleg Gusak for setting up the experimental framework.

\section{References}

[1] S. Baase, Computer Algorithms: Introduction to Design and Analysis, AddisonWesley, Reading, Massachusetts, 1988.

[2] P. Buchholz, An aggregation\disaggregation algorithm for stochastic automata networks, Probability in the Engineering and Informational Sciences 11 (1997) 229-253.

[3] P. Buchholz, Projection methods for the analysis of stochastic automata networks, in: Proceedings of the 3rd International Workshop on the Numerical Solution of Markov Chains, B. Plateau, W. J. Stewart, M. Silva, (Eds.), Prensas Universitarias de Zaragoza, Spain, 1999, pp. 149-168.

[4] R. Chan, W. Ching, Circulant preconditioners for stochastic automata networks, Technical Report 98-05 (143), Department of Mathematics, The Chinese University of Hong Kong, April 1998. 
[5] T. Dayar, Permuting Markov chains to nearly completely decomposable form, Technical Report BU-CEIS-9808, Department of Computer Engineering and Information Science, Bilkent University, Ankara, Turkey, August 1998, available via ftp from ftp://ftp.cs.bilkent.edu.tr/pub/tech-reports/1998/BU-CEIS-9808.ps.z.

[6] T. Dayar, O.I. Pentakalos, A.B. Stephens, Analytical modeling of robotic tape libraries using stochastic automata, Technical Report TR-97-189, CESDIS, NASA/GSFC, Greenbelt, Maryland, January 1997.

[7] T. Dayar, W.J. Stewart, On the effects of using the Grassmann-Taksar-Heyman method in iterative aggregation-disaggregation, SIAM Journal on Scientific Computing 17 (1996) 287-303.

[8] T. Dayar, W. J. Stewart, Comparison of partitioning techniques for two-level iterative solvers on large, sparse Markov chains, SIAM Journal on Scientific Computing 21 (2000) 1691-1705.

[9] P. Fernandes, B. Plateau, W.J. Stewart, Efficient descriptor-vector multiplications in stochastic automata networks, Journal of the ACM 45 (1998) 381-414.

[10] J.-M. Fourneau, Stochastic automata networks: using structural properties to reduce the state space, in: Proceedings of the 3rd International Workshop on the Numerical Solution of Markov Chains, B. Plateau, W. J. Stewart, M. Silva, (Eds.), Prensas Universitarias de Zaragoza, Spain, 1999, pp. 332-334.

[11] J.-M. Fourneau, H. Maisonniaux, N. Pekergin, V. Véque, Performance evaluation of a buffer policy with stochastic automata networks, in: IFIP Workshop on Modelling and Performance Evaluation of ATM Technology, vol. C-15, La Martinique, IFIP Transactions North-Holland, Amsterdam, 1993, pp. 433-451.

[12] J.-M. Fourneau, F. Quessette, Graphs and stochastic automata networks, in: Computations with Markov Chains: Proceedings of the 2nd International Workshop on the Numerical Solution of Markov Chains, W. J. Stewart, (Ed.), Kluwer, Boston, Massachusetts, 1995, pp. 217-235.

[13] O. Gusak, T. Dayar, J.-M. Fourneau, Discrete-time stochastic automata networks and their efficient analysis, submitted to Performance Evaluation, June 2000.

[14] J.R. Kemeny, J.L. Snell, Finite Markov Chains, Van Nostrand, New York, 1960.

[15] J.R. Koury, D.F. McAllister, W. J. Stewart, Iterative methods for computing stationary distributions of nearly completely decomposable Markov chains, SIAM Journal on Algebraic and Discrete Methods 5 (1984), 164-186.

[16] D.F. McAllister, G.W. Stewart, W.J. Stewart, On a Rayleigh-Ritz refinement technique for nearly uncoupled stochastic matrices, Linear Algebra and Its Applications 60 (1984), 1-25.

[17] I. Marek, P. Mayer, Convergence analysis of an iterative aggregation-disaggregation method for computing stationary probability vectors of stochastic matrices, Numerical Linear Algebra with Applications 5 (1998), 253-274.

[18] C.D. Meyer, Stochastic complementation, uncoupling Markov chains, and the theory of nearly reducible systems, SIAM Review 31 (1989) 240-272.

[19] B. Plateau, De l'évaluation du parallélisme et de la synchronisation, Thèse d'état, Université Paris Sud Orsay, 1984.

[20] B. Plateau, On the stochastic structure of parallelism and synchronization models for distributed algorithms, in: Proceedings of the SIGMETRICS Conference on Measurement and Modelling of Computer Systems, Texas, 1985, pp. 147-154. 
[21] B. Plateau, K. Atif, Stochastic automata network for modeling parallel systems, IEEE Transactions on Software Engineering 17 (1991) 1093-1108.

[22] B. Plateau, J.-M. Fourneau, K.-H. Lee, PEPS: A package for solving complex Markov models of parallel systems, in: Modeling Techniques and Tools for Computer Performance Evaluation, R. Puigjaner, D. Ptier, (Eds.), Spain, 1988, pp. 291-305.

[23] B. Plateau, J.-M. Fourneau, A methodology for solving Markov models of parallel systems, Journal of Parallel and Distributed Computing 12 (1991) 370-387.

[24] G.W. Stewart, W.J. Stewart, D.F. McAllister, A two-stage iteration for solving nearly completely decomposable Markov chains, in: Recent Advances in Iterative Methods, IMA Vol. Math. Appl. 60, G. H. Golub, A. Greenbaum, M. Luskin, (Eds.), Springer-Verlag, New York, 1994, pp. 201-216.

[25] W.J. Stewart, Introduction to the Numerical Solution of Markov Chains, Princeton University Press, Princeton, New Jersey, 1994.

[26] W.J. Stewart, K. Atif, B. Plateau, The numerical solution of stochastic automata networks, European Journal of Operational Research 86 (1995) 503-525.

[27] E. Uysal, T. Dayar, Iterative methods based on splittings for stochastic automata networks, European Journal of Operational Research 110 (1998) 166-186.

[28] V. Vèque, J. Ben-Othman, MRAP: A multiservices resource allocation policy for wireless ATM network, Computer Networks and ISDN Systems 29 (1998) 21872200 . 